\title{
Efectos de los comerciales con contenido erótico-sexual sobre el recuerdo, actitudes y actividad fisiológica, en relación con el grado de liberalidad*
}

\author{
Effects of Ads with Erotic-Sexual Content on Recall, Attitudes \\ and Physiological Activity, in Relation to the Degree of Liberality
}

\author{
Marithza SANDOVAL-Escobar ${ }^{\mathrm{a}}$ \\ Fundación Universitaria Konrad Lorenz, Colombia \\ ORCID: http://orcid.org/0000-0001-6551-6938 \\ Jorge Ávila-CAmpos \\ Fundación Universitaria Konrad Lorenz, Colombia \\ PaOla Barreto \\ Fundación Universitaria Konrad Lorenz, Colombia
}

a Autor de correspondencia. Correo electrónico:

marithza.sandoval@konradlorenz.edu.co

Para citar este artículo: Sandoval-Escobar, M., ÁvilaCampos, J., \& Barreto, P. (2018). Efectos de los comerciales con contenido erótico-sexual sobre el recuerdo, actitudes y actividad fisiológica, en relación con el grado de liberalidad. Universitas Psychologica, 17(3), 1-11. https://doi.org/10.11144/Javeriana.upsy1 7-3.ecce

\section{RESUMEN}

El presente estudio pretendió establecer el efecto de comerciales con contenidos de naturaleza erótico-sexual sobre el recuerdo y las actitudes hacia el anuncio y la marca publicitada. Se analizó la activación fisiológica y se buscaron relaciones con el grado de liberalidad-conservadurismo de cada participante. Para ello, se desarrolló un estudio cuasiexperimental con dos grupos, uno conformado por 29 hombres y el otro por 29 mujeres. Se diseñó un programa de televisión que consistía en un documental sobre la naturaleza en donde se insertaron tres tipos de comerciales: A1 (alto contenido erótico-sexual), A2 (bajo contenido erótico-sexual) y A3 (sin contenido erótico-sexual). Los comerciales fueron clasificados de acuerdo con las categorías empleadas por la NICAM y por la ISFP. Se combinaron los órdenes de aparición de estos comerciales y se eligió el orden que recibió cada participante de manera aleatoria. Posteriormente, fueron conectados a un polígrafo que registró su actividad fisiológica frente al programa que observaban. Una vez terminado el procedimiento, se aplicó un cuestionario para medir el recuerdo y actitudes frente al comercial y a la marca. Los resultados muestran que no existen diferencias significativas entre hombres y mujeres en la activación fisiológica frente a los comerciales con alto grado de erotismo y que el contenido eróticosexual aportó significativamente a la recordación de la marca. De la misma manera, no se observaron efectos diferenciales de los contenidos eróticos en relación con el grado de liberalidad de los participantes.

Palabras clave

publicidad erótica; actitudes del consumidor; recuerdo de marca; activación fisiológica.

\section{ABSTRACT}

This study aimed to establish the effect of ad with erotic content of a sexual nature on the recall and attitudes toward the ad and the advertised brand, analyzing the physiological activation of individuals 
and the observed differences between men and women. In addition, the differences in relation to the degree of conservatism of each participant settled. For this, a quasiexperimental study compared two groups, one consisting of 29 men and the other for 29 women developed. Television program was designed consisting in a nature documentary where 3 types of commercial A1 (highly erotic-sexual content), A2 (under erotic-sexual content) and A3 (without erotic-sexual content) were inserted. The commercials were classified according to the categories used by NICAM and the ISFP. The order of appearance of these commercials were combined and the order received each participant was randomly chosen. They were then connected to a polygraph which recorded its physiological activity to the program watching. Once the procedure is completed, a questionnaire was applied to measure memory and attitudes towards trade and off the mark. The results show no significant differences between men and women in physiological arousal against commercial high degree of eroticism and the erotic-sexual content did not contribute significantly improved the brand recall. No differential effects of erotic content depending on the degree of concessionality of participants were observed.

Keywords

erotic advertising; consumer attitudes; brand recall; physiological arousal.

Muchos sectores han enfatizado la necesidad de regular el sexismo en la publicidad, no obstante, no son muchos los estudios disponibles que permitan determinar los efectos del uso de la figura humana en diferentes grados de desnudez y de las escenas eróticas asociadas con las marcas (Nelson \& Paek, 2005, 2008). Los estudios realizados hasta el momento no son concluyentes al respecto y a pesar de las implicaciones sociales que este tipo de anuncios poseen se siguen empleando con alta frecuencia. La participación activa de la mujer en la publicidad es tema de estudio en muchos países, debido a los diferentes estereotipos relacionados con el género, en especial el rol de la mujer (Berganza, García Ortega, \& Grandío-Pérez, 2014; Ford et al., 2015; Halliwell, 2013; Hassan \& Noor, 2016; Raval, 2015).

La publicidad sexista ha sido de gran interés, por lo cual ha sido estudiada inicialmente en Estados Unidos con las primeras investigaciones en relación con los estereotipos de la mujer en la publicidad, de donde se concluye que la representación de la mujer muestra un interés particular por su aspecto físico dentro del ámbito del hogar y pocas veces desempeña un papel profesional (Ceulemans \& Fauconnier, 1980). Dicho interés por el papel de la mujer en la publicidad se ha generalizado a lo largo de los años, como lo demuestran diferentes estudios a nivel mundial asociados no solo a la publicidad, sino a los profundos efectos psicosociales del uso de imágenes físicas estereotipadas (Rosewarne, 2007; Tiggemann \& McGill, 2004; Waller, Fam, \& Erdogan, 2005). El presente estudio desarrolla una de las temáticas más polémicas en la publicidad, como es el efecto de los comerciales con estímulos sexualeseróticos sobre la recordación, las actitudes y la actividad electrofisiológica, tema sobre el cual hay pocas investigaciones.

Desde la década de los setenta, cuando entró en auge la publicidad televisiva y se comenzaron a generar diferentes clases de pauta en radio y televisión, se crea un campo de estudio relacionado con la psicología de la publicidad en el cual se investigan los efectos de diferentes elementos propios del anuncio, el contexto de aparición, los recursos del consumidor, la estructura de la comunicación y el patrón de exposición, ente muchos otros aspectos. Fue en 1981 cuando Mitchell y Olson, a través de una serie de estudios, demostraron que las actitudes hacia un comercial influencian de forma importante la actitud frente a la marca, y que la influencia del aviso está determinada en gran medida por los elementos emocionales y visuales del anuncio. Se postularon diversos mecanismos para esta relación, como por ejemplo, el condicionamiento clásico, las redes asociativas y otros modelos de influencia primaria, que en conjunto predicen que el diseño del estímulo publicitario y su contenido son fundamentales para lograr las ventas de los productos, debido a que las actitudes frente a las marcas parecen estar mediadas por las cogniciones y la intensidad afectiva que genera el comercial (Geuens \& De Pelsmacker, 1999).

Para el caso de los anuncios eróticos, los hombres experimentan sentimientos más favorables frente a ellos, mientras que ante dicha exposición las mujeres muestran reacciones 
afectivas más negativas (Geuens \& De Pelsmacker, 1999). Igualmente, se ha encontrado que la reacción afectiva que provoca el comercial se relaciona con las actitudes y recuerdo de las marcas; los sentimientos positivos, el interés y la alegría se asocian con altos puntajes de reconocimiento del anuncio y de la marca. No obstante, las cogniciones asociadas con la marca poseen una relación limitada con los sentimientos evocados por el anuncio. En el análisis de regresión empleado para identificar la predictibilidad de los sentimientos asociados con el anuncio, Geunes y De Pelsmacker (1998) encontraron que los sentimientos de calidez y humor son los que mejor predicen las actitudes positivas del anuncio y el reconocimiento de marca, no obstante, solo predicen de forma moderada las actitudes ante la marca y la intención de comprarla. En cuanto a las diferencias en las reacciones afectivas frente a diferentes tipos de anuncios, se ha encontrado que no existen diferencias significativas en los sentimientos asociados con comerciales eróticos, humorístico y cálidos. Sin embargo, el interés por el anuncio, la alegría que produce y la falta de irritación ante el mismo, ejerce una influencia positiva sobre las actitudes hacia el anuncio y el reconocimiento de marca para el caso de los anuncios humorísticos, lo cual no se aplica a los comerciales eróticos o no emocionales.

Las diferentes explicaciones a estos efectos se relacionan con el papel que ocupan los sentimientos que evoca el comercial, y por esto la evidencia permite concluir que la estructura y el contenido del anuncio afecta diferencialmente las emociones y cogniciones, dejando un procesamiento diferente para cada una de estas respuestas. Igualmente, los estudios realizados concuerdan en que los comerciales humorísticos, divertidos y eróticos no se pueden clasificar en la misma categoría de comerciales positivos, debido a que cada uno tiene efectos diferentes en las actitudes y en el reconocimiento de marca.

En un estudio inicial, Geunes y De Pelsmacker (1998) realizaron una exposición a diferentes anuncios impresos con contenidos humorísticos, eróticos y afectivos, midiendo en una escala tipo Likert el reconocimiento del aviso y el recuerdo de piezas similares, el reconocimiento de marca, las reacciones cognitivas, la evaluación del grado de humor, calidez y erotismo, las reacciones afectivas inducidas por el aviso, las actitudes frente al aviso, las actitudes hacia la marca y la intención de compra. Los datos confirman que en el caso de los anuncios eróticos los hombres tienden a mostrarse menos irritados y con sentimientos más positivos frente al anuncio, en comparación con las mujeres, no obstante, la reacción es más favorable para ambos sexos que cuando se trata de anuncios no emocionales. Recientemente, Pham, Geunes y De Pelsmacker (2013) seleccionaron una muestra de 1576 consumidores para 1070 comerciales correspondientes a 150 categorías de producto. Los autores demostraron que los sentimientos evocados por el anuncio poseen un importante impacto sobre las evaluaciones de marca, aun en contextos cercanos a las situaciones cotidianas del mercado. Estos efectos sobre las actitudes son tanto directos como indirectos y dependen del involucramiento con la categoría de producto, aun cuando los efectos son más fuertes en productos hedónicos. Esta interacción con la categoría de producto se identificó también en el estudio de Geuens, De Pelsmacker y Faseur (2011), donde se observó que los comerciales emocionales funcionan mejor para algunas categorías, concluyendo que los pobres resultados de algunos comerciales pueden deberse a los productos en sí mismos, más que a los anuncios como tales.

Es claro que, aunque cada vez se use de manera más frecuente la imagen masculina, la mayoría de las veces los estímulos eróticos empleados en la publicidad emplean la figura femenina en diferentes grados de desnudez y la relacionan con muchas categorías de producto. Estos cuerpos, masculinos o femeninos, se muestran perfectos como valor estético y/o sexual, en ocasiones desconectados del contenido y el contexto del comercial (tono de voz, colores, música y voz en off) (Espín López, Marín García, \& Rodríguez Lajo, 2004). Según Tuzla (2012), la imagen sexual y la promesa de satisfacción sexual han 
sido utilizadas de diversas formas durante más de un siglo para vender productos.

En la actualidad, el desnudo, sobretodo el desnudo de la mujer, es utilizado en la publicidad con el objetivo de llamar la atención del consumidor, y se cree que tiene un efecto positivo. No obstante, no se puede generalizar el efecto de la publicidad con estímulos eróticos dado que también las actitudes generales previas moderan los efectos de este tipo de anuncios. Sengupta y Dhal (2008) investigaron las diferencias entre hombres y mujeres en cuanto a su reacción espontánea frente a estímulos sexuales en comerciales y encontraron que cuando existen condiciones limitadas de procesamiento que permiten la generación de la reacción espontánea a nivel visceral, los hombres, en promedio, exhiben una respuesta actitudinal más positiva que las mujeres frente a este tipo de anuncios. Igualmente, se encontró que existen variaciones intragénero relacionadas con las actitudes liberales frente al sexo por parte de las mujeres, quienes reaccionan igual que los hombres frente a los anuncios (les gustan más que los que no tienen estímulos sexuales), cuando son más liberales. Esto sugiere que las normas personales podrían moderar el efecto de los comerciales con estímulos eróticos o sexuales, y que tanto la historia individual como los aspectos culturales se asocian con los efectos de los comerciales eróticos.

En un estudio, Tuzla (2012) indagó acerca de si la presencia de una mujer parcialmente desnuda en publicidad impresa de automóviles en Turquía causaba diferencias en la percepción de la velocidad, valor, atracción y calidad. El investigador reportó que en estudios previos de automóviles se había hallado una mejor evaluación de la atracción, la imagen juvenil y la mejoría en el diseño cuando en la publicidad se presentaba una mujer físicamente atractiva; también señaló que los resultados fueron los mismos para ambos géneros. En el estudio de Tuzla, se eligieron seis automóviles altamente populares y conocidos entre la población, encontrando que la presencia de una mujer parcialmente desnuda genera una diferencia significativa de la percepción de valor solo para tres de los modelos, es decir, que si el vehículo es percibido como económico, la presencia de una mujer parcialmente desnuda en su publicidad mejora las características del mismo, pero que si es percibido como costoso, no hay efecto alguno, dado que producto en sí mismo ya es considerado como atractivo.

Por otra parte, es importante considerar la claridad del mensaje en el que aparecen los estímulos eróticos y la relación que guardan con la marca anunciada. Un aviso publicitario será evaluado más favorablemente cuando el estado afectivo de la persona es positivo que cuando no lo es. Sin embargo, Gorn, Tuan y Yatming (2001) encontraron que ese cambio es más probable cuando el aviso publicitario es ambiguo. Los autores, investigaron la relación entre la valencia y los componentes de la activación de los estados afectivos y el tono afectivo de un anuncio. En un primer estudio, emplearon música para inducir un estado de ánimo placentero o displacentero, mientras que la activación se mantuvo controlada; posteriormente, se presentaron anuncios con un tono afectivo positivo o ambiguo en su tono afectivo. Como se predijo, la valencia del estado afectivo incidió sobre la evaluación del anuncio en una dirección congruente con el estado afectivo previo del individuo. Cuando los avisos eran claramente positivos o negativos y se manipuló la activación, las evaluaciones se polarizaron en la misma dirección (alta activación vs. baja activación), lo cual sucedió con mayor intensidad en las evaluaciones autorreferentes más que en las relativas al objeto. Esto confirma que el proceso que puede afectar las evaluaciones se relaciona con la hipótesis de transferencia de la excitación más que con un proceso atencional propiamente dicho. No obstante, a partir de este interesante estudio, no se pueden extraer conclusiones definitivas puesto que la activación emocional fue medida a través de un cuestionario de autorreporte, el cual bien podría no dar cuenta de la activación emocional real que está experimentando la persona.

Para el caso de la publicidad con estímulos eróticos, la activación fisiológica frente al comercial es mucho más clara y automática, por 
tanto, los hallazgos de los estudios de Gorn, Tuan y Yatming (2001) bien podrían extrapolarse, teniendo en cuenta que la predicción de que su valencia afectiva se puede transferir de manera más expedita a la marca, en el caso de los hombres. Sin embargo, para probar este punto se requiere una medida directa de la activación; los estudios más recientes demuestran que la actividad cerebral y periférica cambia no solo con el género sino también con la categoría de producto, y que estímulos con alta notoriedad en un comercial son una forma de generar atención a otros elementos del anuncio. Las medidas directas de la activación y del proceso de atención que han sido utilizadas en los estudios de consumidor incluyen la tasa cardiaca durante la exposición al contenido, al igual que la medida de la conductancia galvánica de la piel (Bolls, Lang, \& Potter, 2001; Gangadharbatla, Bradley, \& Wise, 2013; Plassmann, Zoëga, Ramsøy, \& Milosavljevic, 2012). La tasa cardiaca se ha tomado principalmente como una medida de atención y la conductancia, como una medida de la activación (arousal) (Kim, Kim, \& Bolls, 2014), y se han usado para evidenciar los efectos del tipo de anuncio y de la imaginería sobre la intención conductual. Así, por ejemplo, Kim et al. probaron anuncios en video vs. anuncios auditivos con alta imaginería, midiendo la tasa cardiaca y la conductancia de la piel durante la exposición a los mismos. No se encontraron diferencias entre los dos tipos de anuncios cuando se medía la tasa cardiaca, pero sí se evidenciaron en la medida de conductancia para los comerciales en video.

El uso de medidas fisiológicas permite que se pueda evaluar de manera directa la valencia de los estímulos para el consumidor. Lo cual es muy importante en el caso de la evaluación de las actitudes frente a los anuncios con estímulos eróticos, debido a que tanto mujeres como hombres pueden verse inhibidos al momento de expresar a través de autorreporte la preferencia por el anuncio y por la marca.

Tradicionalmente, se asume que los avisos con contenidos visuales y verbales de naturaleza erótica poseen un gran poder para generar atención por parte del consumidor y asociaciones positivas frente a las marcas a las que se asocie (Baker \& Churchill, 1977; Boutsikaki \& Balabanis, 2017; Lombardot, 2007; Pope, Voges, $\&$ Brown, 2004). Algunos estudios realizados en la década de los noventa han identificado componentes cognoscitivos y emocionales que se asocian con actitudes positivas hacia el aviso, sugiriendo que el contenido erótico podría provocar una gran activación emocional que se transfiere a la imagen de la marca (Geuens \& De Pelsmacker, 1998). Debido a estas evidencias iniciales, tradicionalmente, se han utilizado estímulos erótico-sexuales en los comerciales principalmente asociados con el cuerpo femenino, con la expectativa de lograr las asociaciones deseadas con la marca. No obstante, no se ha establecido de forma directa si la efectividad de este tipo de anuncios se relaciona con la activación fisiológica que sucede al exponerse a las escenas erótico-sexuales en los comerciales. Igualmente, la evidencia indica que existen variables moderadoras muy importantes de este efecto, como son el sexo del individuo y sus actitudes previas respecto a temáticas relacionadas con la sexualidad, la política, etc. De tal forma, la presente investigación evaluó el efecto de comerciales de naturaleza eróticosexual sobre el recuerdo, las actitudes frente al comercial y la marca publicitada, así como sobre la activación fisiológica, dependiendo del sexo del participante y su grado de liberalidadconservadurismo.

Para efectos de esta investigación, se esperaba encontrar que los hombres mostrarán actitudes más favorables hacia comerciales de alto contenido sexual que las mujeres, que existirán diferencias significativas en las medidas de recuerdo de los tres tipos de comercial a los que se expusieron los participantes, así como entre hombres y mujeres respecto a las actitudes frente a las marcas promocionadas en los comerciales con estímulos erótico-sexuales. De acuerdo a la literatura reportada, se esperaba que las mujeres con actitudes generales más liberales mostraran actitudes más favorables hacia los comerciales erótico-sexuales que las aquellas con actitudes generales como conservadoras, y que esto se pudiera corroborar a partir de diferencias significativas en la activación fisiológica frente 
a cada tipo de comercial al que se exponen los participantes, con una mayor activación frente a los comerciales de naturaleza erótico-sexual (Boutsikaki \& Balabanis, 2017).

\section{Método}

\section{Participantes}

Se incluyeron en el estudio 21 hombres y 29 mujeres con edades comprendidas entre los 18 y 38 años, estudiantes de diferentes carreras de pregrado de diferentes instituciones universitarias. Se realizó una convocatoria durante un mes, y posteriormente se entrevistó a cada persona para asegurar el cumplimiento del perfil requerido. La distribución por edad fue de 18 a 23 años con un porcentaje del $78 \%$ y el $22 \%$ para edades desde los 24 a los 38 años. El nivel socioeconómico al que pertenecían fue del $63 \%$ para el estrato 3 y el $18 \%$ para el estrato 4, mientras que para los estratos 1, 2, 5 y 6 representó el $19 \%$ de la población. El 87.38 $\%$ de los participantes fueron heterosexuales, el $11.65 \%$ de los sujetos manifestó su orientación homosexual y solo el $0.97 \%$, bisexual. El nivel de estudios finalizados por los voluntarios fue del $37.18 \%$ para bachillerato, para técnico o tecnólogo fue del $38.46 \%$, para los estudios universitarios y de postgrado fue de $19.23 \%$ y $5.13 \%$, respectivamente.

\section{Diseño}

Se llevó a cabo un estudio cuasiexperimental con dos grupos de comparación; también se realizaron comparaciones intrasujeto; el sexo del participante se tomó como una variable anidada y se incluyó una covariable consistente en el grado de liberalidad.

\section{Procedimiento}

Se diseñó un programa de televisión consistente en un documental de naturaleza donde se insertaron tres tipos de comerciales: A1 (alto contenido erótico-sexual), A2 (bajo contenido erótico-sexual) y A3 (sin contenido eróticosexual). Los comerciales fueron clasificados de acuerdo con las categorías empleadas por la NICAM (Netherlands Institute for the Classification of Audio-visual Media) y ISFP (Internal Security Fund Police-European Comission for Internal Security Strategy), organizaciones europeas encargadas de la regulación de los contenidos televisivos. Se arreglaron diferentes órdenes de aparición de estos comerciales y se eligió el que recibió cada participante de manera aleatoria. Antes de iniciar el experimento, el participante respondió el cuestionario de datos sociodemográficos y la escala a través de la cual se estableció su grado de liberalidad-conservadurismo. Posteriormente, los participantes fueron conectados a un polígrafo que registró su actividad fisiológica de temperatura y pulso frente al programa que observaban. Una vez terminado el procedimiento, se aplicó un cuestionario para medir recuerdo y actitudes frente al comercial y a la marca.

\section{Instrumentos}

Se emplearon cuatro escalas. La primera se diseñó para realizar una clasificación, mediante calificación de jueces, del grado de erotismo de cada uno de los comerciales empleados en el estudio. Esta escala, denominada Escala de medición del grado de sexualidad explicita y desnudez en la publicidad, incluyó seis dimensiones: desnudez, sexualidad, protagonista, opinión del video, partes del cuerpo e interpretación de las escenas. Para la medición de las actitudes hacia el anuncio y marca, se utilizó la segunda escala de diferencial semántico, adaptada para el estudio a partir de Bigné y Sánchez (2001). La tercera escala de siete ítems tipo Likert se aplicó para establecer el grado de liberalidad-conservadurismo de cada participante. Para esta, los ítems fueron adaptados de la Escala de Autoritarismo de Etchezahar, Prado-Gascó, Jaume y Brussino (2014). El cuarto instrumento consistió en un 
cuestionario con 11 preguntas abiertas para evaluar los contenidos de cada uno de los comerciales y la preferencia por los mismos.

\section{Consideraciones éticas}

La investigación se rigió por las leyes colombianas relacionadas con la práctica profesional de la psicología y sus aspectos éticos, establecidos en el Código Deontológico y Bioético derivado de la Ley 1090 de 2006. En dicho código, se indica la exigencia de lineamientos particulares para asegurar la integridad y dignidad de los participantes en los procesos de investigación. En este sentido, los participantes firmaron un consentimiento informado, mediante el cual se validó su derecho a decidir acerca de dicha participación, como también el compromiso de los investigadores respecto al cuidado y confidencialidad de la información. El procedimiento de la presente investigación se rigió por los principios éticos de respeto, dignidad y bienestar de los participantes, dado que ninguno de los anuncios tuvo contenidos ofensivos, insultantes o pornográficos.

\section{Resultados}

Para realizar el análisis de las diferencias en cada una de las condiciones experimentales, se aplicó una prueba de normalidad que mostró que las actitudes entre los distintos anuncios no cumplen con una distribución de normalidad en relación con el sexo. Por tal razón, se realizó el análisis con la prueba no paramétrica de Kruskall Wallis, y se encontró que únicamente existen diferencias significativas según el sexo para el grupo A1 en el que se presentó alto contenido erótico-sexual (Kruskall-Wallis $=5.430 ; g l=1$; $p=0.02)$. En el grupo de los hombres $(n=$ 21), puntuó más alto en el análisis descriptivo $(\bar{X}=3.214)$ que el grupo de mujeres $(n=$ 29; $\bar{X}=2.693)$. Mientras que para los grupos

A2 y A3 no se obtuvo diferencias significativas $($ Kruskall-Wallis $=1.393 ; g l=1 ; p=0.238$;
Kruskall-Wallis $=0.784 ; g l=1 ; p=0.376)$, respectivamente. En los análisis descriptivos en las mujeres reflejan que para el grupo $\mathrm{A} 2$ y $\mathrm{A} 3$, estos últimos fueron valorados mejor por ellas ( $\bar{X}=2.601 ; \quad \bar{X}=3.647)$. En el caso de los hombres, manteniendo la línea de las mujeres en la evaluación de los anuncios A2 y A3, este último y sin contenido erótico-sexual obtuvieron puntuaciones más altas $(\bar{X}=2.806 ; \quad \bar{X}=$ 3.369).

Para comprobar la relación entre el recuerdo y en nivel de contenido sexual, se evaluaron cuatro tipos de recuerdo, (1) lo que recuerdan haber visto de forma general entre los diferentes anuncios; (2) es un recuerdo relacionado con lo que consideran haber oído y lo que vieron en los diferentes anuncios; (3) hace referencia al recuerdo relacionado con el producto y la marca y (4) el número de personajes que recuerdan que salían en cada uno de los anuncios.

Se realizó ANOVA de medidas repetidas no paramétricas Friedman donde no se evidencian diferencias significativas $(F=1.85 ; p=0.397)$ en cuanto a la recordación, en el número de participantes que salen en los diferentes anuncios. Lo que evidencia que el recuerdo fue similar ante los tres grupos de anuncios (A1, A2 y A3). En cuanto a la puntuación obtenida en las medias en las mujeres, esta fue similar excepto para el grupo de anuncios A3 $(\bar{X}=0.31 ; \bar{X}=$ 0.33; $\bar{X}=0.14$ ); en los hombres el recuerdo fue similar para el A2 y A3, pero no para los anuncios A1 $(\bar{X}=0.19 ; \quad \bar{X}=0.214 ; \quad \bar{X}=0.262)$.

En cuanto a la actitud hacia la marca se realizó un ANOVA de Kruskall Wallis en la que se evidencia que no hay diferencias significativas para los diferentes anuncios eróticos, es decir; que hombres y mujeres tienen actitudes similares. Para el grupo A1, (Kruskal-Wallis $=0.14 ; g l=$ $1 ; p=0.70$ ), para el grupo A2 (Kruskal-Wallis $=0.652 ; g l=1 ; p=0.42)$ y para el grupo A3 sin contenido erótico-sexual (Kruskal-Wallis $=$ 2.417; $g l=1 ; p=0.12$ ). Las mujeres puntuaron más alto para el grupo A3 (3.714), al igual que los 
hombres puntúan más alto para los anuncios tipo A3 ( $\bar{X}=3.357)$, para el grupo A2 las mujeres puntuaron $\bar{X}=2.683$ y los hombres $\bar{X}=$ 2.7. Mientras que para el grupo de anuncios $A 1$, las mujeres obtuvieron una $\bar{X}=2.945$ y los hombres $\bar{X}=3$.

Aun cuando hombres y mujeres evalúan los anuncios de forma similar, cabe preguntarse si todos los anuncios son evaluados de la misma manera. Se realizó la prueba de Friedman que refleja que las mujeres puntúan mejor el tipo de anuncio A3 con una puntuación de $\bar{X}=$ 3.71 y puntuando más bajo los anuncios A2 con una $\bar{X}=2.68$ y para el grupo de anuncio A1 con una $\bar{X}=2.95$ los hombres al igual que las mujeres puntuaron los anuncios del grupo A3 más alto con una puntuación de $\bar{X}=3.36$, el grupo A2 con una $\bar{X}=2.7$ y el A1 con una puntuación de $\bar{X}=3$.

Para probar la hipótesis relacionada con la dimensión liberalismo-conservadurismo, se calculó una correlación de Spearman para establecer el grado de relación entre el grado de liberalismo o conservadurismo con las actitudes hacia los anuncios. Se observa que el grado de liberalismo-conservadurismo no guarda relación con la actitud hacia el anuncio (liberalismo/ conservadurismo A1 $p=0.568$; A2 $p=0.835$; A $3 p=0.22$ y para liberalismo/conservadurismo $\mathrm{A} 1 p=0.796 ; \mathrm{A} 2 p=0.854 ; \mathrm{A} 3 p=0.995)$. Se aprecia en las mujeres que a medida que mejora la actitud hacia el anuncio A1, también lo hace la actitud hacia el anuncio A2. Mientras que la actitud del anuncio A3 no tiene nada que ver con los anteriores. En cambio, las puntuaciones obtenidas en los hombres no guardan relación, es decir; si puntúan bajo en A1 y A2 no se sabe cómo van a puntuar en $\mathrm{A} 3$; se asumen como puntuaciones independientes. Los estadísticos descriptivos reflejan que las mujeres obtuvieron puntuaciones más bajas en los anuncios de tipo
A1 a diferencia de los tipos A3, por su parte, los hombres puntuaron los distintos tipos de anuncios de una forma similar.

Los resultados, asociados a la activación fisiológica frente a cada tipo de comercial al que se exponen los participantes, indican que la presencia de los diferentes estímulos no genera cambios estadísticamente significativos en la activación fisiológica $(F=0.337, p=0.686)$. Al igual que en el pulso $(F=0.137, p=0.791)$ ante los distintos anuncios (A1, A2 y A3). Estos resultados evidencian que la exposición a los anuncios erótico-sexual no producen variaciones fácilmente perceptibles a nivel fisiológico.

\section{Discusión}

En esta investigación se evaluaron el efecto de comerciales de naturaleza erótico-sexual sobre el recuerdo, las actitudes frente al comercial y la marca publicitada, así como sobre la activación fisiológica, teniendo en cuenta factores como el sexo del participante y su grado de liberalidadconservadurismo. Se observó que los hombres tienen una actitud más positiva ante los estímulos con alto contenido erótico-sexual, lo cual es coherente con las investigaciones de Dianoux y Linhart (2010), de LaTour y Henthorne (1993) y de Sengupta y Dahl (2008). En las mujeres no se encontraron diferencias significativas en ninguno de los grupos A1, A2 y A3, aunque existe una tendencia hacia una actitud más positiva frente al comercial cuando el contenido erótico-sexual es más bajo. Cabe aclarar que dicha actitud ha reportado cambios al comparar diferentes generaciones en otras culturas, mostrando que algunas mujeres, en la actualidad, se sienten menos ofendidas por el contenido sexual de los anuncios (Zimmerman \& Dahlberg, 2008).

Los resultados de la presente investigación evidencian que no existen diferencias significativas en el recuerdo en relación con el tipo de comercial A1, A2 y A3. No obstante, existe una tendencia en las mujeres a recordar menos personajes de los comerciales con bajo contenido sexual, mientras que los hombres 
recuerdan menos personajes de los anuncios con alto contenido sexual.

Igualmente, no se encuentran diferencias significativas entre hombres y mujeres en cuanto a su actitud hacia la marca, lo que contradice algunos estudios culturales que han mostrado que el contenido sexual resulta en una actitud menos favorable hacia la marca en el caso de las mujeres (Dahl, Sengupta, \& Vohs, 2009; Dianoux \& Linhart, 2010; LaTour \& Henthorne, 1993; Wolin, 2003); sin embargo, son pocos los estudios disponibles que evalúen esas actitudes en población latinoamericana y en comparación con otras culturas.

Estos resultados son congruentes con las puntuaciones en la escala de liberalidad empleada en el estudio. Se encontró que el grado de liberalismo-conservadurismo no guarda relación con la evaluación de la actitud hacia el anuncio y que no existe una relación entre la activación fisiológica y el tipo de anuncio. De tal modo, el estudio sugiere que las personas son poco sensibles al contenido sexual de los anuncios y que esta actitud es independiente del género. Lo que sí reflejan las medidas de tendencia central es que tanto hombres como mujeres evaluaron mejor los anuncios del grupo A3 (sin contenido erótico-sexual).

Los hallazgos del presente trabajo tienen implicaciones importantes en dos sentidos. En primera instancia, permiten afirmar que no existe un efecto diferencial de los comerciales con contenido erótico sobre las actitudes y el recuerdo de la marca, en comparación con aquellos comerciales cuyo contenido es informativo o emocional. Esta evidencia desafía la creencia de publicistas y comunicadores relacionada con la necesidad de involucrar escenas de contenido erótico y exposiciones del cuerpo femenino como un medio para mejorar el reconocimiento de las marcas. En segundo lugar, indica que los contenidos eróticos en el comercial no generan efectos fisiológicos particulares en las personas y que dichas reacciones son independientes del sexo del individuo. Esto también es una fuerte evidencia en contra de la creencia difundida según la cual los efectos de los comerciales sobre las actitudes y creencias de los consumidores están mediados por la reacción fisiológica frente a las escenas eróticas. Es importante ampliar los estudios que evalúen en distintos niveles la forma en la que los consumidores latinoamericanos perciben la publicidad asociada a contenido sexual, teniendo en cuenta diferentes variables no incluidas en este estudio como la edad, región geográfica, grado de estudio, percepción hacia la violencia sexual y de género, entre otras. También se deben hacer estudios que comparen estas variables en distintos países de la región y los contrasten con los estudios reportados en la presente investigación.

\section{Referencias}

Baker, M. J., \& Churchill, G. A., Jr. (1977). The impact of physically attractive models on advertising evaluations. Journal of Marketing Research, 14, 538-555. https://do i.org/10.2307/3151194

Berganza, R., García Ortega, C., \& Grandío Pérez, M. D. M. (2014). Women and advertising: Evolution of stereotypes in Spanish mass media. Proyecto "Observatory of Advertising in Navarra". Navarra, ES: Universidad de Navarra, Instituto Navarro de la Mujer.

Bigné Alcañiz, J. E., \& Sánchez García, J. (2001). Antecedentes y efectos de la actitud hacia el anuncio. Propuesta de un modelo de variables latentes. Investigaciones Europeas de Dirección y Economía de la Empresa, 7(2), 41-62. Recuperado de https://dialnet.unirio ja.es/servlet/articulo?codigo $=187794$

Bolls, P. D., Lang, A., \& Potter, R. F. (2001). The effects of message valence and listener arousal on attention, memory, and facial muscular responses to radio advertisements. Communication Research, 28(5), 627-651. https://doi.org/10.1177/00 9365001028005003

Boutsikaki, K., \& Balabanis, G. (2017). A new typology on sex appeal advertisements. En C. L. Campbell (Ed.), The customer is not always right? Marketing orientations 
in a dynamic business world. Developments in Marketing Science. Proceedings of the Academy of Marketing Science (pp. 219-223). Nueva York: Springer. https://do i.org/10.1007/978-3-319-50008-9_60

Ceulemans, M., \& Fauconnier, G. (1980). Imagen, papel y condición de la mujer en los medios de comunicación social: compilación y análisis de los documentos de investigación. París: Unesco.

Dahl, D. W., Sengupta, J., \& Vohs, K. D. (2009). Sex in advertising: Gender differences and the role of relationship commitment. Journal of Consumer Research, 36(2), 215-231. https://doi.org/10.1086/59 7158

Dianoux, C., \& Linhart, Z. (2010). The effectiveness of female nudity in advertising in three European countries. International Marketing Review, 27(5), 562-578. https://d oi.org/10.1108/02651331011076590

Espín López, J., Marín García, M. \& Rodríguez Lajo, M. (2004). Análisis del sexismo en la publicidad. Revista de Investigación Educativa, 22(1), 203-231.

Etchezahar, E., Prado-Gascó, V., Jaume, L., \& Brussino, S. (2014). Validación argentina de la Escala de Orientación a la Dominancia Social. Revista Latinoamericana de Psicología, 46(1), 35-43. https://doi.org/1 0.1016/S0120-0534(14)70004-4

Ford, J. B., Honeycutt, E. D., Jr., LaTour, M. S., Voli, P. K., Joseph, M., \& Job, J. (2015). A Comparison of American and New Zealand consumer perceptions of sex role portrayals in advertising. En M. Levy \& D. Grewal (Eds.), Proceedings of the 1993 Academy of Marketing Science (AMS) Annual Conference (pp. 154-158). Nueva York: Springer.

Gangadharbatla, H., Bradley, S., \& Wise, W. (2013). Psychophysiological responses to background brand placements in video games. Journal of Advertising, 42(2-3), 251-263. https://doi.org/10.1080/00913367 .2013 .775800

Geuens, M., \& De Pelsmacker, P. (1998). Feelings evoked by warm, erotic, humorous or non-emotional print advertisements for alcoholic beverages. Academy of Marketing Science Review, 19(1), 1-31. Recuperado de http://www.amsreview.org/articles/geue ns01-1998.pdf

Geuens, M., \& De Pelsmacker, P. (1999). Affect intensity revisited: Individual differences and the communication effects of different types of emotional stimuli. Psychology $\mathbb{E}$ Marketing, 16(3), 195-209. https://doi.org/10.1002/ (SICI) 1520-6793(199905) 16:3<195::AIDMAR1 > 3.0.CO;2-Z

Geuens, M., De Pelsmacker, P., \& Faseur, T. (2011). Emotional advertising: Revisiting the role of product category. Journal of Business Research, 64(4), 418-426. https://d oi.org/10.1016/j.jbusres.2010.03.001

Gorn, G., Tuan, M., \& Yatming, L. (2001). When Arousal Influences Ad Evaluation and Valence Does Not (and Vice Versa). Journal of Consumer Psychology, 11 (1), 44-55. https ://doi.org/10.1207/S15327663JCP1101_4

Halliwell, E. (2013). The impact of thin idealized media images on body satisfaction: Does body appreciation protect women from negative effects? Body Image, 10(4), 509-514. https://doi.org/10.1016/j.bodyim. 2013.07.004

Hassan, R. \& Noor, S. (2016). Portrayal of Women in National and International Advertisements in Pakistani Magazines: Why Need to Rethink. Journal of Marketing, 27, 81-91. Recuperado de http://iiste.org/Journals/index.php/JMC R/article/view/33121/34020

Kim, S., Kim, D., \& Bolls, P. (2014). Tourist mental-imagery processing: Attention and arousal. Annals of Tourism Research, 45, 63-76. https://doi.org/10.1016/j.annals.201 3.12 .005

LaTour, M. S., \& Henthorne, T. L. (1993). Female nudity: Attitudes towards the ad and the brand, and implications for advertising strategy. Journal of Consumer Marketing, 10(3), 25-32. https://doi.org/10.1108/0736 3769310041956 
Lombardot, E. (2007). Nudity in advertising: What influence on attention-getting and brand recall? Recherche et Applications en Marketing [English Edition], 22(4), 23-41. https://doi.org/10.1177/2051570707 02200401

Mitchell, A., \& Olson, J. (1981). Are product attribute beliefs the only mediator of advertising effects on brand attitude. Journal of Marketing Research, 18(3), 318-332. https://doi.org/10.2307/3150973

Nelson, M. R., \& Paek, H. J. (2005). Crosscultural differences in sexual advertising content in a transnational women's magazine. Sex Roles, 53(5-6), 371-383. http s://doi.org/10.1007/s11199-005-6760-5

Nelson, M. R., \& Paek, H. J. (2008). Nudity of female and male models in primetime TV advertising across seven countries. International Journal of Advertising, 27(5), 715-744. https://doi.org/10.2501/S0265048 708080281

Pham, T., Geuens, M., \& De Pelsmacker, P. (2013). The influence of ad-evoked feelings on brand evaluations: Empirical generalizations from consumer responses to more than 1000 TV commercials. International Journal of Research in Marketing, 30(4), 383-394. https://doi.org/1 0.1016/j.ijresmar.2013.04.004

Plassmann, H., Zoëga Ramsøy, T., \& Milosavljevic, M. (2012). Branding the brain: A critical review and outlook. Journal of Consumer Psychology, 22, 18-36. https://d oi.org/10.1016/j.jcps.2011.11.010

Pope, N. K. L., Voges, K. E., \& Brown, M. R. (2004). The effect of provocation in the form of mild erotica on attitude to the ad and corporate image: Differences between cause-related and product-based advertising. Journal of Advertising, 33(1), 69-82. https://doi.org/10.1080/00913367.2 004.10639154

Raval, M. B. (2015). Discrimination of women in Indian advertisement in the age of neoliberal economy. Paripex: Indian Journal of Research, 4(12), 225-227.
Rosewarne, L. (2007). Pin-ups in public space: Sexist outdoor advertising as sexual harassment. Women's Studies International Forum, 30(4), 313-325. https://doi.org/10.1 016/j.wsif.2007.05.003

Sengupta, J., \& Dhal, D. W. (2008). Gender-related reactions to gratuitous sex appeals in advertising. Journal of Consumer Psychology, 18(1), 62-78. https://doi.org/10. 1016/j.jcps.2007.10.010

Tiggemann, M., \& McGill, B. (2004). The role of social comparison in the effect of magazine advertisements on women's mood and body dissatisfaction [Número especial]. Journal of Social E Clinical Psychology, 23(1), 23-44. h ttps://doi.org/10.1521/jscp.23.1.23.26991

Tuzla, H. (2012). Does Sex in Print-Ads Affect Perceived Product Specifications in Conservative Societies?: the Case of Turkey. Procedia- Social and Behavioral Sciences, 62 (24), 1133-1137. https://doi.org/10.1016 /j.sbspro.2012.09.194

Waller, D., Fam, K., \& Erdogan, Z. (2005). Advertising of controversial products: A cross-cultural study. Journal of Consumer Marketing, 22(1), 6-13. https://doi.org/10.1 108/07363760510576509

Wolin, L. D. (2003). Gender issues in advertising: An oversight synthesis of research: 1970-2002. Journal of Advertising Research, 43(1), 111-130. https://doi.org/10 .2501/JAR-43-1-111-130

Zimmerman, A. \& Dahlberg, J. (2008). The Sexual Objectification of Women in Advertising: A Contemporary Cultural Perspective. Journal of Advertising Research, 48(1), 71-79. https://doi.org/10.2501/S002 1849908080094

\section{Notas}

* Artículo de investigación. 